\title{
FDTD Method for the Analysis of the EM Field from a Moving Source
}

\author{
Shafrida Sahrani", Tatsuya Akata", and Michiko Kuroda** \\ "Faculty of Engineering, Universiti Malaysia Sarawak, Malaysia \\ "Graduate School of Bionics, Computer and Media Sciences, Tokyo University of Technology, Japan \\ ${ }^{* *}$ School of Computer Science, Tokyo University of Technology, Japan \\ sshafrida@feng.unimas.my
}

\begin{abstract}
A numerical technique for the analysis of the EM field by a moving source or a moving body can be significantly important for the realization of new optical/nanotechnology devices. We have previously proposed the Overset Grid Generation method coupled with FDTD method for the analysis of the EM field with moving boundaries considering Doppler Effect. By overlapping one moving sub-mesh on a static main mesh, each mesh is calculated alternately by using interpolation technique. For higher velocity value, Lorentz transformation is applied to the FDTD method. In this paper, this technique is proposed for solving the EM field when the input source is moving. As a bench mark, the received wave at the observation point is calculated when the source moves for $x$-direction in free space intersect at right angle by the dielectric object. The numerical results are compared with the stationary case and the moving case.
\end{abstract}

Index Terms - FDTD method, Lorentz transformation, moving boundary problems, moving source, Overset Grid Generation method.

\section{INTRODUCTION}

In recent years, the analysis of the electromagnetic (EM) field with moving boundary has received much attention in the literature [1],[2]. This analysis would certainly be of great interest with the development of the new and efficient modeling techniques. The Overset Grid Generation method combined with Finite-Difference Time-Domain (FDTD) method and Lorentz transformation has been previously proposed to solve the EM field from a moving body for higher velocity cases [3],[4]. In this paper, the proposed technique is applied for the analysis of the EM field with the presence of a moving source.

The Overset Grid Generation method has been used mainly in the computational fluid dynamics (CFD) [5]. This method consists of a static main mesh and a moving sub-mesh that overlapped each another. It can reduce the geometrically complex problem to a simple set of grids. The proposed numerical technique aims to find a solution to keep the submesh moving with high velocity value. According to the special relativity, Lorentz transformation requires at least two frames of reference that move with relative velocity [6]. In this paper, Lorentz transformation is reinforced to the Overset Grid Generation method for the case of the moving body with high velocity value.

The FDTD method is widely used to solve the EM field problems due to its low computational complexity [7]. Moreover, this method is very easy to implement in any computing environment including on the structured grids. The time components for the FDTD method are obtained at alternate half-time steps. However, the time component in Lorentz transformation changes at each grid point. Here, the time components that were changed in Lorentz transformation are fixed by using linear interpolation scheme in the Overset Grid Generation method. This allows a coherent point in time component with the FDTD method, which is an important element of this proposed numerical technique.

In this paper, this proposed Overset Grid Generation method combined with the FDTD method and Lorentz transformation is applied to the analysis of the EM field when the input source is moving. As a bench mark, the received wave at the observation point is calculated when the source moves for $\mathrm{x}$-direction in free space intersect at right angle by the dielectric object. The numerical results of the moving case are compared with the stationary case.

\section{LORENTZ TRANSFORMATION FOR FDTD METHOD}

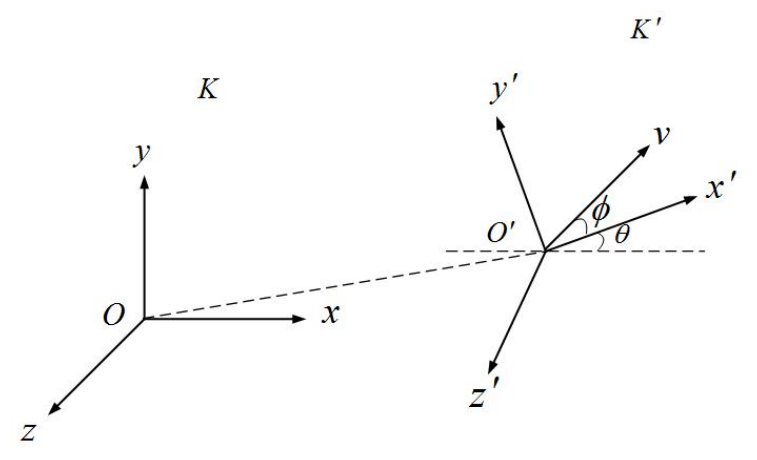

Fig. 1. Two inertial frames in relative motion.

Fig. 1 shows the basic Lorentz transformation in two inertial frames and move relative to one another [6]. Consider two observers, $O$ and $O^{\prime}$ in frame $K$ and $K^{\prime}$ starts at the initial time $t=t^{\prime}=0$, respectively. The observer $O^{\prime}$ in $\left(x^{\prime}, y^{\prime}, z^{\prime}, t^{\prime}\right)$ axis moves with angular $\theta$ against the observer $O$ in $(x, y, z, t)$ axis with relative velocity, $\mathbf{v}(\mathrm{m} / \mathrm{s})$. When the observer $O^{\prime}$ reaches time $t^{\prime}$, Lorentz transformation can be described as in Eq. (1) and Eq. (2) [6]. 